\title{
La construcción del sistema comunitario de salud de Guarjila: sistematización de una experiencia de Atención Primaria de Salud Integral en El Salvador durante el período 1987-2007
}

Construction of the community health system in Guarjila: systematization of an experience of Comprehensive Primary Health Care in El Salvador in the period 1987-2007

María Argelia Dubón Abrego', Dagoberto Menjívar López², Eduardo Espinoza Fiallos ${ }^{3}$, Christa Baatz

\footnotetext{
' Doctora en Medicina pela Universidad de El Salvador; Directora del Primer Nivel de Atención del Ministerio de Salud - San Salvador, El Salvador.

argeliadubon@yahoo.es
}

2 Doctor en Medicina; Coordinador de la MicroRed de Salud de Guarjila y de la Unidad Comunitaria de Salud Familiar y Especializada de Guarjila - Guarjila, El Salvador.dagomenjivar@yahoo.com

${ }^{3}$ Doctor en Medicina, Master en Salud Pública; Ex-decano de la Facultad de Medicina de la Universidad de El Salvador; Docente-Investigador en Políticas de Salud de la Maestría de Salud Pública de la Universidad de El Salvador; Viceministro de Políticas de Salud, Ministerio de Salud (MINSAL) El Salvador.

eespinoza@salud.gob.sv

${ }^{4}$ Doctora en Medicina. cbaatz2005@yahoo.de
RESUMEN Se sistematizó una experiencia de salud comunitaria, surgida durante la Guerra Civil en El Salvador, en un contexto de represión institucionalizada, describiendo el proceso desarrollado con elementos de la Atención Primaria de Salud integral, tomando como ejes el proceso histórico de construcción, el empoderamiento social y los indicadores de impacto. La población abordó intersectorialmente sus problemas, mejorando condiciones básicas como vivienda, agua, letrinas y energía eléctrica; produjo sus alimentos; y creó un modelo de educación popular y de salud con agentes comunitarios. Las intervenciones en salud progresaron desde la atención y prevención de la enfermedad, hasta un abordaje integral de determinantes sociales. La organización, la participación y el trabajo intersectorial fueron claves para mejorar la salud y el desarrollo integral de la comunidad.

PALABRAS CLAVE: Salud comunitaria; Empoderamiento; Intersectorialidad; Determinantes sociales.

\begin{abstract}
A community health experience during the Civil War in El Salvador was systematized, which emerged in a context of institutionalized repression, describing the process developed as a comprehensive Primary Health Care. The axes of systematization were the processes of historical construction experience, social empowerment, and indicators of impact on community health. The population addressed their problems across inter-sectoral actions, improving basic conditions such as housing, water, latrines, and electricity. Population also produced their own food, created a model of popular education and health based in community agents. Health interventions evolved from the original focus in the injury to the prevention of disease and later to a comprehensive approach to social health determinants. The community organization, participation and inter-sectoral actions were keys to improve health and comprehensive development of the community.
\end{abstract}

KEYWORDS: Community health; Empowerment; Intersectoral actions; Social determinants of health. 


\section{Introducción}

Se investigó una experiencia de salud comunitaria, surgida en la década de los 1980, durante la Guerra Civil contra la dictadura militar, en una de las zonas mayormente conflictivas en el Norte de El Salvador, específicamente en el departamento de Chalatenango, que fue un importante escenario de organización de las comunidades y fuerzas revolucionarias.

Los objetivos de la investigación fueron: sistematizar la experiencia de salud comunitaria en Guarjila, El Salvador, durante el período de 1987 al 2007, reflexionando sobre los procesos de construcción de la experiencia para extraer lecciones que contribuyan a revitalizar la estrategia de Atención Primaria de Salud (APS) Integral; conocer las características de los pobladores de la comunidad; identificar los factores facilitadores y limitantes del contexto que incidieron en la construcción de la experiencia; describir los procesos de organización, participación comunitaria, toma de decisiones, intersectorialidad, distribución de responsabilidades, equidad, sostenibilidad y contraloría social así como los indicadores de impacto; y por último, evidenciar factores facilitadores y limitantes para el desarrollo de la experiencia y su incidencia en el Sistema Nacional de Salud, identificando las lecciones que pueden contribuir a la revitalización de la APS Integral.

La investigación fue un estudio cualitativo de sistematización de experiencias, aplicado a salud comunitaria, con tres ejes de sistematización: el proceso histórico de construcción, el empoderamiento comunitario, y los indicadores de impacto. Se definieron preguntas generadoras enmarcadas en los ejes de sistematización, identificando categorías y sub-categorías de análisis. No fue necesario un período de inmersión porque los investigadores residían en la comunidad investigada desde hace más de 30 años. El levantamiento de los datos se realizó a través de entrevistas a profundidad a actores claves, grupos focales, acción-observación-participante, revisiones bibliográfica y documental.

\section{Antecedentes}

El Salvador estuvo inmerso en un conflicto político y social desde la década de los años 1970, que tuvo como base un amplio movimiento obrero, campesino, estudiantil y profesional, lo cual culminó con la Guerra Civil en los años 1980, después que los espacios democráticos fueron anulados por la dictadura militar y las demandas sociales fueron brutalmente reprimidas. Esta guerra popular terminó con la firma de los acuerdos de paz en el 1992 y el país entró en un largo proceso de readaptación. En aquella época, se empezaron a abrir espacios democráticos antes negados a la población por la élite política y militar. El Frente Farabundo Martí para la Liberación Nacional (FMLN) máximo representante de la clase trabajadora que lideró el levantamiento armado contra la dictadura militar se convirtió en partido político, empezó a competir en los procesos electorales y a ganar sistemáticamente diputaciones y alcaldías a nivel nacional.

En El Salvador no se conocían experiencias de salud comunitaria antes de la Declaración de Alma Ata; existían algunos servicios asistenciales administrados por la iglesia, pero que no estaban enmarcados en la APS. Después de la declaración, los gobiernos de entonces implementaron programas verticales y selectivos de alto impacto como inmunización, lactancia materna, rehidratación oral, control del crecimiento, y planificación familiar.

El Departamento de Chalatenango, ubicado al Norte del país en la frontera con Honduras, es el departamento más grande del país con $1.957 \mathrm{~km} 2$, de los menos poblados y uno de los cinco más pobres, con comunidades mayormente marginadas y servicios básicos precarios.

La región Nor-Oriental del departamento donde se ubica la comunidad de estudio comprende varios de los municipios catalogados como de extrema pobreza, y fue uno de los escenarios más fuertes del pasado conflicto armado salvadoreño. Al comparar los índices de pobreza nacional y el departamento, se observó que la incidencia de pobreza nacional total es del $43 \%$ (el $38 \%$ urbana y el $53 \%$ rural). Chalatenango representa el 3,3\% de la población nacional (201.702 habitantes) y para 2008, el 23,6\% de su población se encontraba en pobreza extrema y el $33,4 \%$ en pobreza relativa con un total de pobreza del $57 \%$, por encima del promedio nacional (43\%) para el mismo año (PNUD, 2010). Por lo tanto, ubicándose como el cuarto departamento con 
más pobreza extrema y el quinto con mayor pobreza total (MINISTERIO DE ECONOMÍA, 2010).

La región es montañosa con tierras pedregosas y difícilmente cultivables por la baja fertilidad. Las cosechas de maíz, fríjol y arroz nunca han alcanzado para la subsistencia, por eso los pobladores tuvieron que migrar internamente en forma periódica para trabajar como jornaleros en otras partes del país. Por otra parte, se da simultáneamente una alta migración poblacional hacia el exterior, especialmente hacia los Estados Unidos, cuyo gobierno deporta continuamente inmigrantes salvadoreños a pesar de lo cual se mantiene el flujo migratorio ilegal principalmente de jóvenes que lo perciben casi como la única posibilidad de mejorar sus condiciones de vida.

La comunidad de Guarjila es un cantón de la zona rural que pertenece al municipio de Chalatenango, ubicado a $8 \mathrm{~km}$ al Oriente de la ciudad, tiene una población de 1.762 habitantes de la cual más de la mitad son mujeres. Al igual que otras comunidades pobres y rurales del país, en su mayoría subsisten de la agricultura, ganadería, y remesas familiares.

\section{Período del conflicto}

La población de Guarjila y otras comunidades de la zona participaron en el movimiento social organizado durante el levantamiento popular entre el período 1980-1992 contra la tiranía militar en el país.

Muchas de estas comunidades adquirieron experiencia organizativa durante las luchas reivindicativas de los ańos 1970, posibilitando a los campesinos del Nor-oriente de Chalatenango autogobernar sus pequeñas comunidades cuando el gobierno se retiró de la zona, resolviendo problemas vitales como salud, educación, alimentación y otras necesidades cotidianas con los pocos recursos que tenían.

Baatz y Orellana (1998) relatan cómo surgió la salud comunitaria en estas comunidades en medio de la Guerra Civil, durante los años 1980. Argumentan que las raíces del desarrollo del sistema de salud popular de la zona Nor-oriental del departamento de Chalatenango se deben buscar en el proceso de concientización y organización de las masas campesinas (comunidades organizadas) que se inició en los años 1960 y fue desarrollándose en los 1970s (BAATZ; ORELLANA, 1998).

En 1998, una investigación desarrollada con apoyo del Programa INCO de la Unión Europea identificó en El Salvador 86 experiencias locales innovadoras en salud (ELIS), las cuales implementaban en grado variable la APS Integral, entre ellas la experiencia de salud comunitaria de Guarjila (NIJMEJEN INSTITUTE FOR INTERNATIONAL HEALTH et al, 2001), siendo objeto de estudio de tesis de grado y postgrados en varias ocasiones (BARTEN; ESPINOZA, 1998; ESPINOZA; ELLIAS; VILLALTA, 2000).

En 2006, la Organización Panamericana de Salud (OPS) confirió al equipo de salud de Guarjila el reconocimiento de "Héroes de todos los días", por su importante labor y sus logros en el mejoramiento de la salud de la comunidad basada en atención integral, según los principios de la APS Integral.

\section{Materiales y Métodos}

\section{Tipo de estudio}

Estudio cualitativo de tipo sistematización de experiencias, aplicado a la salud comunitaria.

\section{Población de estudio}

El estudio se realizó en la comunidad de Guarjila, con la participación de los habitantes de la comunidad. Se reconstruyó la memoria histórica del proceso de construcción y desarrollo del sistema de salud comunitario.

\section{Objeto de estudio}

Se sistematizó la experiencia de salud de la comunidad con énfasis en el proceso histórico de construcción, en los procesos organizacionales, y en los contextos político, económico, social y cultural en el que se ha desarrollado; se identificaron elementos relacionados con la APS Integral.

\section{Marco muestral}

Para la sistematización de la experiencia, fueron seleccionados actores y grupos claves comunitarios involucrados en el proceso, además se realizaron revisiones 
documental y bibliográfica de fuentes comunitarias y externas.

\section{Ejes de sistematización}

Se definieron tres ejes de sistematización: el proceso histórico de construcción de la experiencia de salud comunitaria; los procesos comunitarios de empoderamiento; y los indicadores de impacto.

\section{Preguntas de la investigación}

- ¿Cuál fue el contexto en el que se construyó la experiencia de salud en la comunidad de Guarjila entre el 1987 y el 2007?

- ¿Cúales son las características esenciales de la comunidad, qué cambios hay en sus condiciones de vida, y cuáles perciben respecto a su salud durante estos 20 años?

- ¿Cúales fueron los procesos de organización y participación social en la construcción de la experiencia de salud comunitaria?

- ¿Cuáles fueron los factores facilitadores y limitantes en los procesos desarrollados?

- ¿Qué momentos fueron decisivos y favorecieron la experiencia de salud comunitaria para mantenerse en el tiempo?

- ¿Qué ha facilitado o limitado a la experiencia de salud comunitaria para que incida en el Sistema Nacional de Salud?

- ¿Cuáles son las evidencias que permiten demostrar que la experiencia de salud comunitaria está enmarcada dentro de la APS Integral?

\section{Técnicas de obtención de las informaciones}

Entrevistas a profundidad a actores claves, grupos focales, observación-acción-participante, revisiones documental y bibliográfica y de material audiovisual.

\section{Fuentes de información}

Primarias fueron compuestas por líderes y lideresas de la comunidad, grupos organizados, equipos de salud y organismos de dirección comunitaria. Las secundarias fueron documentos de la experiencia de salud comunitaria, planes operativos, informes, investigaciones previas, audiovisuales y publicaciones que hacen referencia a las comunidades en la postguerra salvadoreña.

\section{Resultados}

La población de Guarjila estuvo refugiada en el refugio de Mesa Grande Honduras desde el 1981 hasta el 1987, que albergaba a más de 11.000 refugiados. Retornaron a la comunidad en octubre de 1987 con el apoyo de organizaciones sociales, organizaciones no gubernamentales (ONGs), la Iglesia Católica, otras iglesias solidarias y organismos internacionales, entre ellos el Alto Comisionado de las Naciones Unidas para los Refugiados (ACNUR), el Comité Internacional de la Cruz Roja (CICR) y Médicos sin Fronteras.

La mayoría de las comunidades repobladas entre 1987 y 1990 organizó equipos de promotores/as de salud que fueron capacitados/as en los refugios por Médicos sin Fronteras. Estos promotores atendieron las necesidades básicas de atención de salud y las lesiones ocasionadas por la guerra a la población, tanto en el refugio como al llegar a las comunidades.

En ese contexto, la comunidad de Guarjila construyó una infraestructura sanitaria básica y organizó un equipo de salud con mayor capacidad que el restante de las comunidades, convirtiéndose en un centro de referencia para la red de salud comunitaria de las comunidades de la zona Nor-oriente de Chalatenango durante la guerra y posteriormente a los acuerdos de paz en el 1992.

A mediados del 1993, el Ministerio de Salud instaló paulatinamente puestos de salud administrados por personal ajeno a la comunidad, desplazando gradualmente de la zona a los promotores de salud comunitaria que por muchos años habían trabajado por la salud de las comunidades. La población de Guarjila rechazó las pretensiones del Ministerio de Salud de absorber el sistema comunitario de salud a su red de servicios, porque privilegiaba el abordaje curativo y marginaba la participación social y los demás elementos de la APS Integral. 
La experiencia en Guarjila fue construida por la comunidad en un contexto adverso durante el conflicto armado salvadoreño. La organización de la comunidad se evidenció a través de una directiva comunal, máxima representación organizada de la comunidad, por equipos y comités locales de salud, educación, agua, agricultura, mujeres, jóvenes, lisiados de guerra, etc.

La población se organizó para mejorar progresivamente sus condiciones de vida elementales: construyó el sistema de agua potable, las viviendas y letrinas; desarrolló su propio sistema de producción de alimentos y creó un modelo de educación con maestros populares y un modelo de salud con agentes comunitarios de salud, principalmente promotoras de salud y rehabilitación, parteras y grupos de mujeres voluntarias.

La organización comunitaria permitió abordar intersectorialmente la problemática comunitaria e involucrar en la toma de decisiones a la mayoría de la comunidad. La solidaridad y la búsqueda constante de la equidad se evidencian en los diferentes procesos comunitarios.

La comunidad se involucró activamente en las distintas intervenciones dirigidas por el equipo de salud, asumió la experiencia en salud como propia y la defendió ante los múltiples intentos de cooptación por agentes externos incluido el estado.

El trabajo del equipo de salud se ha modificado progresivamente, partiendo de una atención medicalizada centrada en el tratamiento de la enfermedad y lesiones de guerra, posteriormente incorporó la prevención y la promoción de la salud, hasta llegar a un abordaje con enfoque de género, derechos humanos, y determinantes sociales.

El trabajo comunitario se evidencia en una amplia infraestructura comunitaria construida por la gente, entre los que destacan una amplia escuela que alberga a más de 500 alumnos, un centro de salud comunitario que incluye a la Clínica 'Ana Manganaro' y uno de rehabilitación, la casa comunal, carpintería, tienda y comedor, casas para talleres, casa-museo y radio comunitaria. También fue construido por ellos el sistema de agua potable que aun continúa bajo administración local. Todas estas construcciones fueron financiadas con fondos de cooperantes extranjeros, pero la comunidad aportó todo el trabajo. La inversión estatal a nivel nacional y regional fue muy reducida.

Como resultados de estas iniciativas comunitarias, las mejoras en las condiciones de vida se evidencian en el $94 \%$ de las familias que disfrutan viviendas propias construidas de materiales duraderos con servicios básicos: agua potable intra-domiciliar, letrinas y energía eléctrica; habitaciones y servicios estos construidos con trabajo comunitario.

Los principales resultados en la salud percibidos por la población se enmarcan dentro de la prevención, la educación en salud y la adopción de conductas saludables, especialmente en saneamiento básico e higiene personal, tratamiento de agua de consumo humano, reducción y tratamiento de enfermedades gastrointestinales y parasitarias, infecciones respiratorias, infecciones de transmisión sexual, disminución de la automedicación, consulta temprana y continua y mejoras en los hábitos alimenticios.

Entre los indicadores que miden el impacto en la salud de la acción comunitaria se destaca en primer lugar la ausencia de mortalidad infantil y materna desde 1997. La atención prenatal es del 100\% de las embarazadas, controladas en el establecimiento y el domicilio, con un promedio de seis controles intra-murales. La inscripción prenatal precoz (antes de las 12 semanas) es del $90,5 \%$ y un $100 \%$ de parto institucional: todos los partos son atendidos en un centro hospitalario de segundo nivel.

La atención infantil fue ampliada en el 100\% de niños menores de cinco años acompañados en la estrategia de Atención Integrada a las Enfermedades Prevalentes de la Infancia (AIEPI), con esquema de vacunación completo según la edad. Además, no se encontró desnutrición en los menores de cinco años al momento de levantamiento de datos. Respecto a la planificación familiar, el $40 \%$ de las mujeres en edad fértil usan algún método temporal o permanente. La tasa bruta de natalidad es de 14.4/1.000 habitantes, inferior al promedio nacional de 20/1.000 habitantes y la mitad de la que corresponde al departamental de 31/1.000 habitantes.

Los factores que limitaron la construcción de la experiencia fueron: el aislamiento sistemático de la comunidad por las diferentes instancias del estado, la 
discriminación de los trabajadores de salud comunitarios por los trabajadores de salud del gobierno, y la dependencia del financiamiento de la solidaridad internacional. Todos los factores obligaron a la comunidad a dar un soporte financiero que limitó el acceso universal. Aun así y a pesar de carecer de una remuneración adecuada, el equipo de salud mantuvo su compromiso con la gente con pocas deserciones.

\section{Discusión}

La experiencia de salud construida por la comunidad de Guarjila es un esfuerzo amplio de sus habitantes que a través de la organización, la participación comunitaria, y el trabajo intersectorial permitieron el empoderamiento de sus pobladores y se convirtieron en elementos principales para el desarrollo de la experiencia de salud, constituyendo un determinante fundamental para el desarrollo integral de la comunidad.

Es evidente que las experiencias organizativa y participativa acumuladas durante el levantamiento popular permitieron a los pobladores un alto nivel de conciencia y concebir la comunidad como un proyecto colectivo, permitiendo mantener los proyectos comunitarios, especialmente la salud. Por otra parte, el equipo de salud, predominantemente local, mantuvo el compromiso y protagonismo convirtiéndose en agentes de transformación de la realidad de la comunidad, incidiendo positivamente en la población para mejorar los indicadores de salud. Esto denota la importancia de que el equipo de salud viva y trabaje en su comunidad. El abordaje intersectorial, entendido como la acción de los diferentes grupos organizados que componen la comunidad, permitió un mayor involucramiento de esta.

Las intervenciones en salud, que al inicio estaban centradas en la enfermedad, progresaron a la prevención y a la promoción de la salud, hasta un abordaje integral con enfoque de género, derechos humanos y determinantes de la salud, denotando el dinamismo comunitario.

\section{Conclusiones}

La experiencia de salud construida fue un esfuerzo amplio de la comunidad donde la organización, la participación comunitaria, y el trabajo intersectorial permitieron el empoderamiento de la población, constituyendo los elementos principales para el avance de la experiencia de salud; simultáneamente con otros determinantes del desarrollo integral de la comunidad.

El trabajo del equipo de salud avanzó progresivamente de la atención centrada en la enfermedad, a la promoción de la salud y la acción sobre los determinantes sociales. El equipo de salud fue protagónico convirtiéndose en agentes de cambio para la transformación de las causas injustas que afectan a la comunidad. Los indicadores actuales de salud evidencian el éxito de la experiencia, y su relevancia se refleja en el actual proceso de reforma de salud en El Salvador, que ha tomado como modelo esta experiencia.

La sistematización de la experiencia de salud de Guarjila demuestra que es posible trabajar en pro de la APS Integral como fue concebida originalmente, la que está inmersa en el desarrollo integral de la comunidad; que la salud ha sido un componente fundamental del desarrollo, estimulando el papel protagónico de la comunidad y elevando su nivel de 'conciencia social' (OMS, 1978).

El equipo de salud compuesto por los agentes locales permitió un mayor compromiso de la comunidad. Las mujeres han tenido un papel importante en el desarrollo socioeconómico superando la inequidad de género.

Guarjila demostró que la comunidad organizada tiene mayor capacidad resolutiva, y por consiguiente mayores posibilidades de desarrollo social; igualmente que los proyectos tienen éxito siempre y cuando la comunidad sea partícipe en su diseño, gestión, y desarrollo.

\section{Agradecimientos}

A la Iniciativa de Investigación en Salud Mundial, una asociación dedicada al financiamiento de la investigación entre los Institutos Canadienses de Investigación 
en Salud, la Agencia Canadiense para el Desarrollo Internacional, el Ministerio de Salud de Canadá, el Centro Internacional de Investigaciones para el Desarrollo, y la Agencia de Salud Pública de Canadá. También a la comunidad de Guarjila, por su contribución en la reconstrucción de su experiencia de salud comunitaria; a los agentes comunitarios de salud por su aporte y protagonismo en el desarrollo de esta experiencia; y a la doctora María Isabel Rodríguez por su constante motivación a la investigación.

\section{Referências}

BAATZ, C.; ORELLANA, M. Capacidad resolutiva de comunidades rurales que cuentan con promotores de salud en Chalatenango y Morazán. San Salvador: El Salvador, 1998.

BARTEN F.; ESPINOZA E. Rescue of Local Experiences in El Salvador and Nicaragua for the Health Sector Reform Process. European Commission Contract Holders'Meeting. Downing College, Cambridge, UK. 29 March-1 April 1998.

ESPINOZA, E.A., ELÍAS M.A. Y VILLALTA V. EI SILOS de la Zona Norte de San Salvador 1992-1997: Una experiencia innovadora en salud. Tesis para optar al grado de Maestría en Salud Pública. Facultad de Medicina, Universidad de El Salvador; San Salvador; 2000.

MINISTERIO DE ECONOMÍA EL SALVADOR. Indicadores de Calidad. 2012. Disponible en: <http://www.elsalvadorcompite.gob.sv/ portal/page/portal/ESV/Pg_Sise_II>. Acceso en: 20 mar. 2012.

NIJMEJEN INSTITUTE FOR INTERNATIONAL HEALTH; CENTRO DE INVESTIGACIONES Y ESTUDIOS DE LA SALUD DE NICARAGUA et al.
Rescate de Experiencias Locales Innovadoras en Salud en El Salvador, Guatemala y Nicaragua durante el periodo 1980 - 1995" financiado por la Unión Europea (Programa INCO-DEV; Contract number IC18*CT960058); Nijmen: 2001. Mimeo.

ORGANIZACIÓN MUNDIAL DE LA SALUD (OMS). Declaración de Alma Ata. Atención Primaria de Salud. OMS: Ginebra, 1978.

PROGRAMA DAS NAÇÕES UNIDAS PARA O DESENVOLVIMENTO (PNUD). Informe de Desarrollo Humano 2010. San Salvador: El Salvador, 2010.

Recibido para publicação em Abril/2012

Versão definitiva em Agosto/2012

Suporte financiero: Programa de investigación en Salud Global Teasdale-Corti

- un programa de investigación

Conflito de interesse: inexistente 